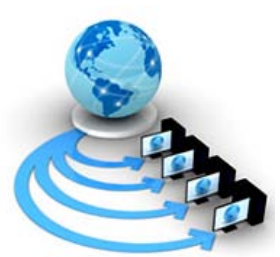

Volume 8, No. 9,November-December 2017

International Journal of Advanced Research in Computer Science

RESEARCH PAPER

\author{
Available Online at www.ijarcs.info
}

\title{
IMPLEMENTATION OF ENHANCED NA GAUNN TECHNIQUE (ENGT) FOR RESOURCE BLOCK ALLOCATION IN LTE SYSTEMS FOR IMPROVING THROUGHPUT
}

\author{
Kanwaldeep Kaur \\ Department of Computer Science \&Engg \\ Sri Sai College of Engg and Technology, \\ Manawala (Amritsar) Punjab (India)
}

\author{
Rimmy Chuchra \\ Department of Computer Science \&Engg \\ Sri Sai College of Engg and Technology, \\ Manawala (Amritsar) Punjab (India)
}

\begin{abstract}
The study shows that distinct and unique features viz. high speed data rate, self-organized network, packet optimized radio access low latency and flexible bandwidth deployment are the parameters that enable LTE (Long Term Evolution) to be preferred in wireless communication systems. This terminology is preferred especially for mobile nodes that help to improve speed that ultimately increases throughput. As LTE (Long Term Evolution) uses Orthogonal Frequency Division Multiplexing (OFDM) for the downlink data transmission that means for sending data over narrow band barriers $5 \mathrm{MHz}$ career bandwidth is used. The complete working of LTE is based on the basic principle of OFDM (Orthogonal Frequency Division Multiplexing). At the first step, it divides the broad spectrum into multiple narrow bands and transmits information on this narrow channel in parallel.OFDM meets the LTE requirement for spectrum flexibility and enables costefficient solutions for very wide carriers with high peak rates. Orthogonal frequency-division multiplexing (OFDM), is a frequency-division multiplexing (FDM) scheme used as a digital multi-carrier modulation method for implementing scheduling. In general, scheduling plays a vital role on the time of resource allocation and that only can be based on the need as well as priority of the user. The main three parameters considerations are viz. resource block scheduling, power control and client association taken by the authors. The mainmotive to design and implement this new designed methodology is the right utilization of resources at a right time when users send request to the system that either may send on the same time or send on the different-2 time slots. By implementing this ENGT (Enhanced Na Gaunn Technique) speed of LTE systems can be easily improved that automatically helps to improve the throughput of the system. In this paper, there are 4 different types of modulation coding schemes (MCS)are applied as like QPSK, QAM,16QAM and 256 QAM etc. The main focus of authors is while implementing ENGT (Enhanced Na Gaunn technique) improves the throughput of the system. By implementing this, resources are properly distributed in resource blocks at specific data rate requirement and allocated resource rate \& rightly utilized at right time. The main benefit to implement this ENGT isto handle user requests efficiently while performing right distribution of resources at right time. Hence, authors said this enhanced version of Na Gaunn algorithm may help to fulfill requests optimally and especially on time without any delay after sending the request to the computer system. In this way, the throughput of the system can be easily improved by implementing Enhanced Na Gaunn Technique and automatically the channel quality indicator and performance would automatically be improved.
\end{abstract}

Keywords: Long term Evolution, wireless communication, throughput, resource allocation techniques, security, scheduling algorithms, modulation and coding schemes and OFDM(Orthogonal Frequency Division Multiplexing).

\section{INTRODUCTION}

As the dependency of users on the internet is going to be increases day by day because of most of the tasks are completed very quickly and easily. By utilizing several windows software users can communicate with the computer systems. The operating system is act like an interface between the user and the computer system. So, professionals say operating systems plays a vital role in the digital world where an operating system is kind of low level software that support a computer basic functions such as scheduling tasks and controlling peripherals. In addition, operating system also performs most common functions as an example storage management, memory management and process management [8]. Instead of all these functions operating system may also performs many more functions but scheduling is one of the most important function of operating system where scheduling is the process in which operating system arrange or plan for some particular jobs and events which one is execute first on timely basis. There is one exceptional case if two or more jobs or events are entering in same time of operating system then which one event is handling first or execute first? Without any delay. So, for solving such type of problems operating system designers designed several algorithms and mechanisms are run synchronously and events will be handled on time. As authors studied in several papers, there are number of scheduling algorithms are used now a days as an example shortest job first, first comes first serve, priority scheduling, shortest remaining time, round robin scheduling, multiple level scheduling [1] and maximum channel indicator algorithm [2][7] etc. Each algorithm has its own pros and cons. So, professionals say no any specific algorithm gives hundred percent perfect output in every aspect. And still, experts are continuing facing throughput [5] and channel quality problems. So, to overcome this better throughput problem authors implemented a new methodology for scheduling ENGT that is termed as Enhanced Na Gaunn Technique [6] that gives good throughput with better channel quality hence the performance of the computer system will be automatically improved.

In this research paper, ENGT is implemented. This new designed methodology uses 4 different types of modulation techniques viz. QPSK16 QAM, 64 QAM, 256 QAM etc while entering the number of users uses given number of segments. The number of users as well as the number of 
segments may be increase or decrease that depends on the requirement of the users. The main motive to implement this new designed methodology ENGT [6] is to increase the overall throughput of the system without any delay during the execution of the tasks or events. The main benefit to utilize this ENGT [6] tasks or events are scheduled timely without any delay and users will also collect output on time. In this way, the concept of waiting queue of jobs or events will be slowly- 2 removed. In this way, this new designed methodology also contribute for improving the speed and capacity of 4G technology of LTE (long Term Evolution) systems[2][3]. Hence, the problem of resource allocation [4][1] at a right time can be solved.

\section{RESEARCH DESIGN}

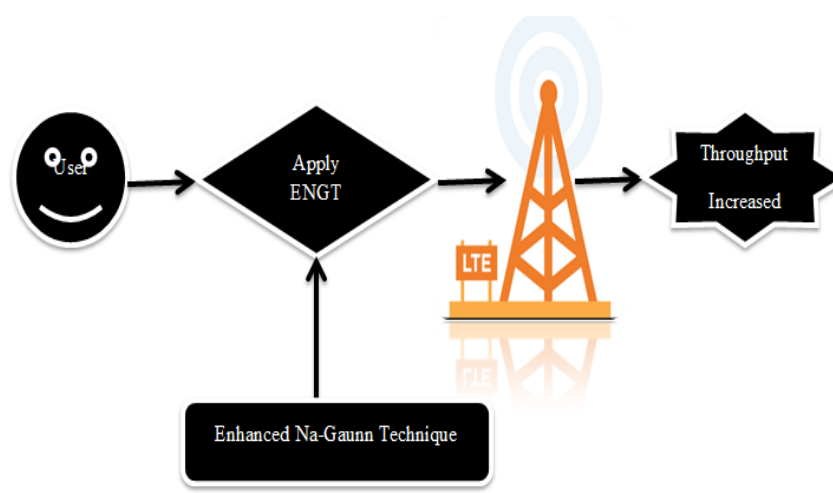

Figure.2: Communication between User and LTE Systems.

III Implementation of ENGT(Enhanced Na Gaunn Technique)

Step-1) Initialize number of users and assign hypothetical values for different Segment Blocks.

\begin{tabular}{|c|c|c|c|c|c|c|c|}
\hline & SB1 & SB2 & SB3 & SB4 & SB5 & SB6 & SB7 \\
\hline User1 & 1 & 1.5 & 2 & 2.5 & 0.5 & 1 & 0.5 \\
\hline User 2 & 2.5 & 0.5 & 1 & 2 & 1.5 & 1.5 & 1 \\
\hline User 3 & 1.5 & 1 & 0.75 & 2 & 1.5 & 1.75 & 1.5 \\
\hline User 4 & 1.5 & 1 & 1.5 & 2 & 1 & 1.5 & 1 \\
\hline
\end{tabular}

Table.1: CQI Matrix [Users: 4, Segment Blocks: 7]. Step 2) Initialize hypothetical values of Rate required and Rate Allocated.

\begin{tabular}{|c|c|c|}
\hline & Rate & Rate \\
Required & Allocated \\
\hline User 1 & 1.0234 & 1.0752 \\
\hline User 2 & 2.34 & 1.008 \\
\hline User 3 & 3.324 & 0 \\
\hline User 4 & 0.765 & 1.3306 \\
\hline
\end{tabular}

Table.2: Required rate and Allocated Rate of ENGT.

Step-3)Apply ENGT for three different users and choose three different choices [1].

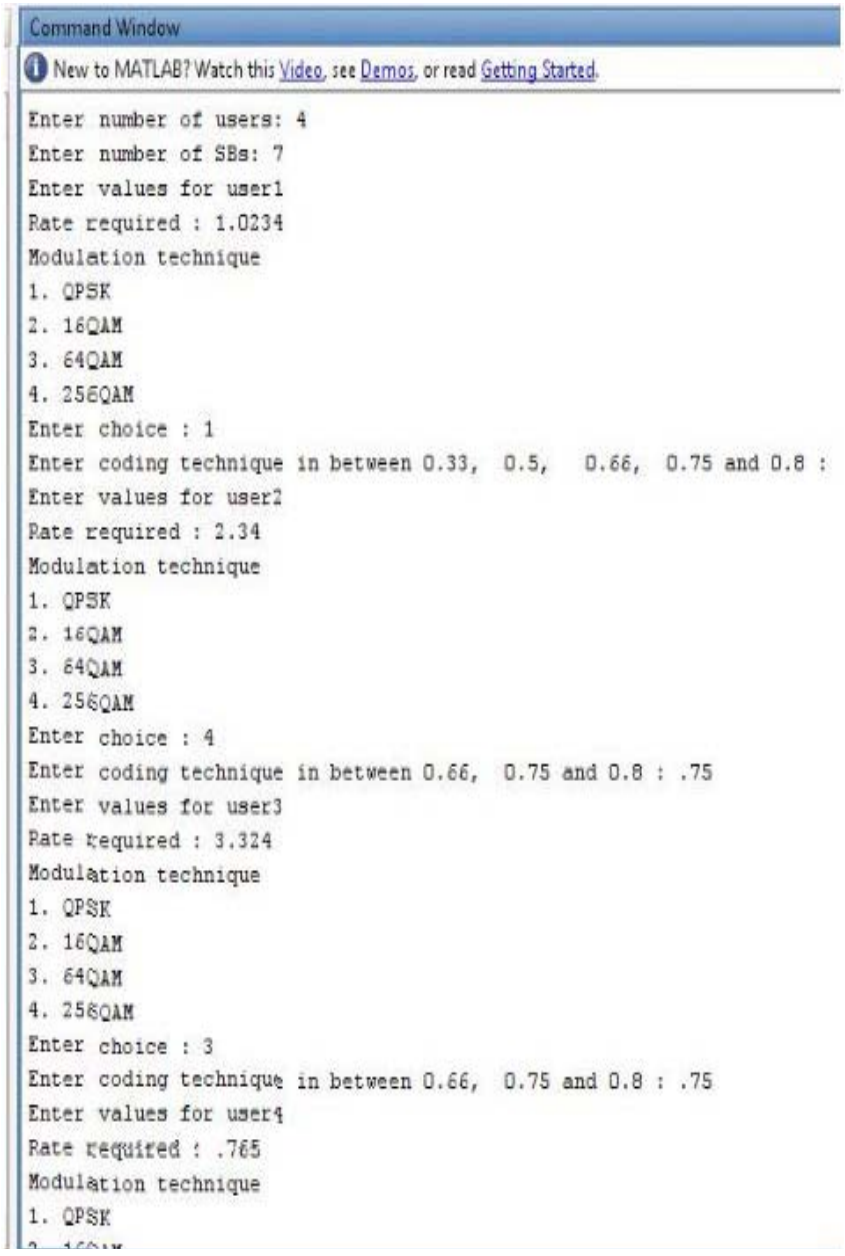

Step-4) Then Set Priorities for Assigned hypothetical values for ENGT. 


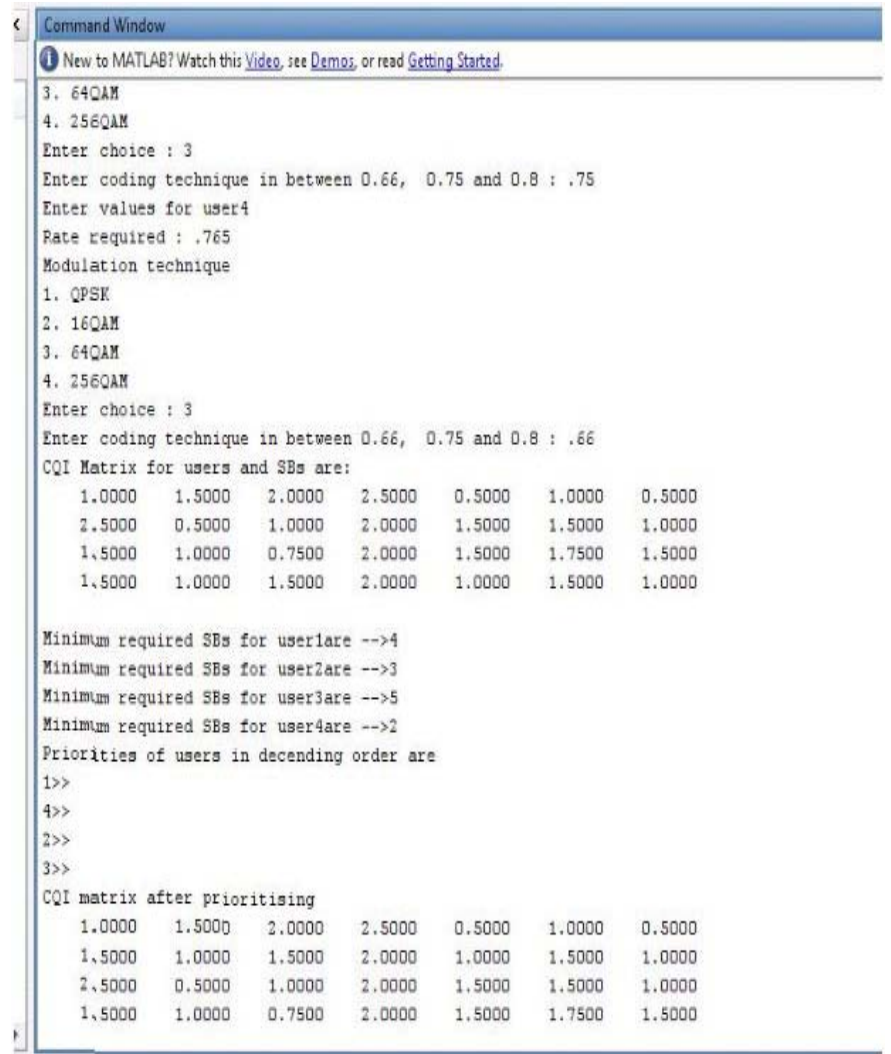

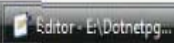

Step-5)After Prioritizing Design CQI Matrix.

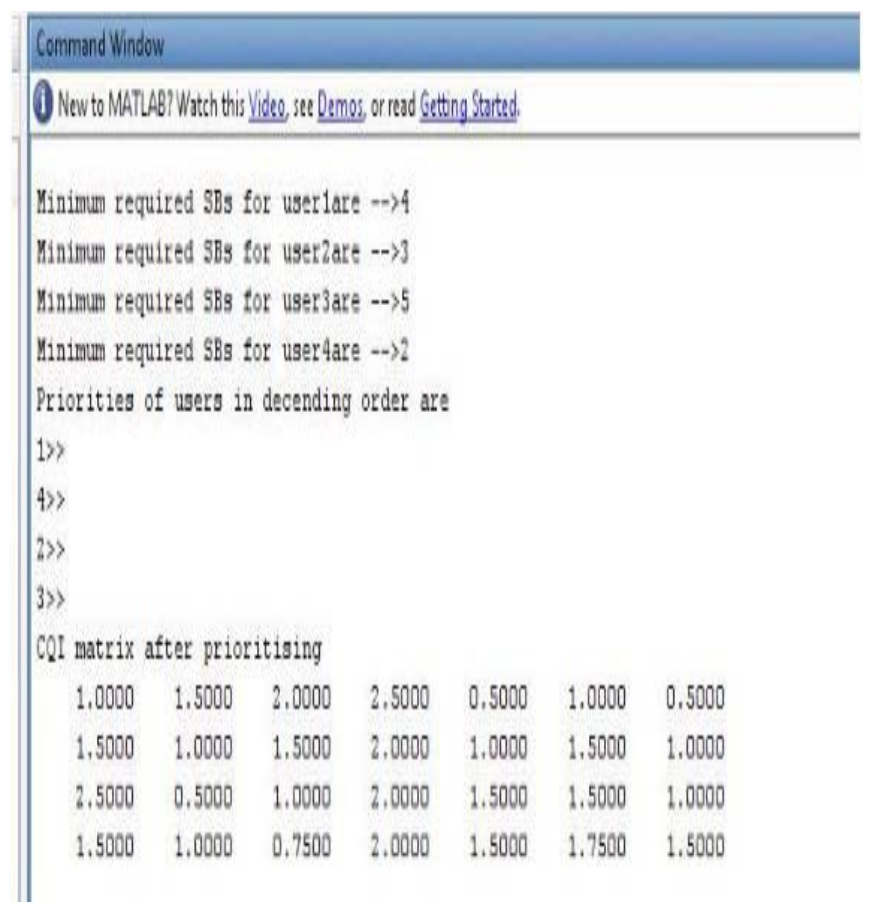

Step-6) Enter Filled rate of 4 Users \&Calculate throughput. // on the basis of time taken for receiving output.

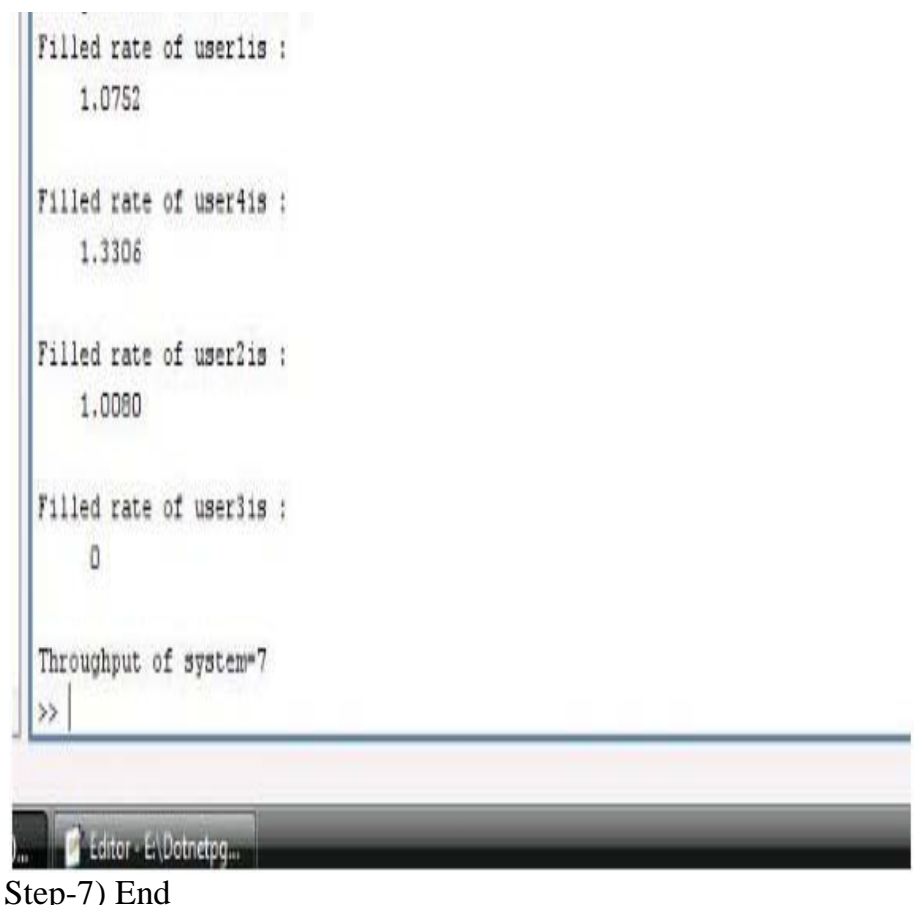

Step-7) End

\section{CONCLUSION}

In this paper, authors implemented new proposed methodology in thesis work whose named is "Enhanced Na Gaunn technique for resource block allocation in LTE (Long Term Evolution) systems that helps to improve speed as well as level of security. The rate requirement for CQI (Channel Quality Indicator) matrix should be given in the form of input. The condition during implementation is resources are allocated to particular user who got segment block first \& the minimum required SBs (Segment Blocks) are calculated for each users on the basis of prioritization of users.On the basis of that prioritization available resource blocks are allocated to users and allocated rate of each user and throughput of system is calculated.

\section{FUTURE WORK}

In future, this work will be extended by considering different-2 algorithms of resource allocation scheduling in LTE (Long term Evolution) systems for improving throughput. The number of segment blocks and users may increase for achieving more accuracy in throughput. In addition, one more distinct feature of optimal resource allocation may add in this work. This additional feature may facilitate users to get easy way to get resource block.

\section{REFERENCES}

[1]Honghai Zhang, Narayan Prasad and SampathRangarajan"MIMO Downlink Scheduling in LTE systems" in 2012, IEEE.

[2]Aderemi A. Atayero, "An Introduction to LTE". 3GPP LTE Encyclopedia. Retrieved December 3, 2010.

M. Faulkner,"Long Term Evolution (LTE): A Technical Overview". Motorola. Retrieved July 3, 2010. 
[3] GPP. UTRA-UTRAN long term evolution (LTE) and 3GPP system architecture evolution (SAE). http://www.3gpp.org/article/lte, 2010.

[4] Mohsen M. Tantawy, Adly S. Tag Eldien, and RokaiaMounirZaki 1, "A Novel Cross-Layer Scheduling Algorithm for Long Term-Evolution (LTE) Wireless System", Canadian Journal on Multimedia and Wireless Networks, Vol. 2, No. 4, December 2011.

[5] http://en.wikipedia.org/wiki/matlab

[6]Kanwaldeepkaur and RimmyChuchra,” Proposing Enhanced Na Gaunn Technique for Resource Block Allocation in
LTE (Long Term Evolution) Systems for improving Quality of Service”, International Journal of Advance Research and Computer Science”,Vol.8,No.7,July-Aug 2017.

[7] Y. Su and L. Ming, "Backhaul Resource Allocation in LTEAdvanced Relaying Systems”, IEEE Wireless Communications and Networking Conference: MAC and Cross-Layer Design, pp- 1207-1211, 2012.

[8]https://www.tutorialspoint.com/operating-system/os-processscheduling-algorithms.htm. 